\title{
Design of Non-Fragile State Estimators for Discrete Time-Delayed Neural Networks with Parameter Uncertainties
}

\author{
Yajing Yu, Hongli Dong*, Zidong Wang, Weijian Ren and Fuad E. Alsaadi
}

\begin{abstract}
This paper is concerned with the problem of designing a non-fragile state estimator for a class of uncertain discrete-time neural networks with time-delays. The norm-bounded parameter uncertainties enter into all the system matrices, and the network output is of a general type that contains both linear and nonlinear parts. The additive variation of the estimator gain is taken into account that reflects the possible implementation error of the neuron state estimator. The aim of the addressed problem is to design a state estimator such that the estimation performance is non-fragile against the gain variations and also robust against the parameter uncertainties. Sufficient conditions are presented to guarantee the existence of the desired non-fragile state estimators by using the Lyapunov stability theory and the explicit expression of the desired estimators is given in terms of the solution to a linear matrix inequality. Finally, a numerical example is given to demonstrate the effectiveness of the proposed design approach.
\end{abstract}

\section{Index Terms}

Discrete-time neural networks; Time-delayed neural networks; State estimation; Non-fragile state estimator; Uncertain systems.

\section{INTRODUCTION}

The past two decades have witnessed a surge of interest on both theoretical investigations and algorithm developments of the recurrent neural networks (RNNs) due mainly to their remarkable ability to exhibit dynamic temporal behavior in order to extract/detect/approximate functional information from complicated or imprecise data. So far, the RNNs have come to play a more and more important role in a variety of application areas including pattern recognition, image processing, optimization calculation and so on. On

This work was supported in part by the National Natural Science Foundation of China under Grants 61422301 and 61374127, the Outstanding Youth Science Foundation of Heilongjiang Province under Grant JC2015016, the Technology Foundation for Selected Overseas Chinese Scholar from the Ministry of Personnel of China, and the Alexander von Humboldt Foundation of Germany.

Y. Yu, H. Dong and W. Ren are with the College of Electrical and Information Engineering, Northeast Petroleum University, Daqing 163318, China. (Email: shiningdhl@vip.126.com)

Z. Wang is with the Department of Computer Science, Brunel University London, Uxbridge, Middlesex, UB8 3PH, United Kingdom. He is also with the Faculty of Engineering, King Abdulaziz University, Jeddah 21589, Saudi Arabia. (Email: Zidong.Wang@brunel.ac.uk)

F. E. Alsaadi is with the Faculty of Engineering, King Abdulaziz University, Jeddah 21589, Saudi Arabia.

* Corresponding author. 
the other hand, since early 90s, the time-delay has been recognized as a ubiquitous phenomenon that could cause undesired oscillations or even instability in both biological and man-made neural networks [2]. As an active research branch, the study on the dynamical behaviors of RNN with time-delays has recently attracted an ever-increasing interest from many communities including neural science, signal processing and control engineering. Accordingly, in the past years, a large number of results have been available in the literature on the dynamics analysis issues (e.g. stability, synchronization and estimation) for RNNs with various kinds of time-delays such as constant, time-varying, discrete, distributed or mixed delays, see [1], [6], [11], [13], [18], [22], [28] for some representative works. Very recently, in [21], the passivity analysis problem has been investigated for a class of switched neural networks subject to stochastic disturbances and time-varying delays by using the average dwell-time approach. In [33], the adaptive synchronization problem has been addressed for memristor-based neural networks with time-varying delays by virtue of the differential equation theory with discontinuous right-hand sides.

In many practical applications, the actual values of the neural states of a RNN are vitally important. For example, in optimization problems, the RNNs can be implemented physically in designated hardware such as application-specific integrated circuits where the optimization is carried out in a truly parallel and distributed manner. In this case, the neuron states are closely related to the equilibria as well as the decision-making solutions. However, because of the large scale of the RNN as well as the implementation cost, the neuron states are often not fully observable and only the network outputs are available that contain partial information about the network states. As such, accurate estimation of the neuron states through measured outputs becomes an essential prerequisite for successful accomplishment of certain tasks such as approximation and optimization by using RNNs. In [34], the problem of state estimation has been first proposed for neural networks with time-varying delays. Since then, such a problem has received considerable research attention for both continuous- and discrete-time neural networks, see e.g. [15], [17], [23], [25], [30], [34], [35].

As is well known, the parameter uncertainties are often unavoidable in real systems due to modeling inaccuracies and/or changes in the environment. In recent years, a great deal of effort has been devoted to the robustness analysis for uncertain systems [7], [12], [16], [29]. Despite the rich body of literature on the state estimation issues for RNN with parameter uncertainties, most results obtained so far have been based on the assumption that the desired state estimator can be realized precisely. Such an assumption is, however, not necessarily true in certain engineering practice. When implementing a state estimator digitally, the implementation errors are often inevitable due probably to analogue-to-digital conversion, rounding errors, finite precision or internal noise. As discussed in [19], a small or even tiny drift/fluctuation/error with the parameter implementation of the designed controller/estimator could lead to unexpected fragility of the closed-loop system as a whole. In other words, the parameters of the actually implemented controller/estimator might have slight deviations from their expected values, and therefore designed controller/estimator should have certain degree of tolerance or non-fragility against the possible parameter deviations. In the past decade, the problem of non-fragile control has gained much attention with respect to the implementation errors in controllers/estimators [24], [27], [32], [36], [37]. However, when it comes 
to the discrete-time RNNs with time-delays, the non-fragile state estimation problem has not been fully studied yet, not to mention the case where the uncertainties also enter into other network parameters. It is, therefore, the main purpose of this paper to shorten such a gap.

Motivated by the above discussion, we aim to design a non-fragile state estimator for a class of discretetime neural networks with parameter uncertainties. A sufficient condition for the asymptotic stability of the error dynamics of the state estimation is obtained and the gain matrix of the state estimator is derived by solving a linear matrix inequality (LMI). A numerical example is presented to demonstrate the effectiveness of the theoretical results obtained. The main contribution of this paper lies mainly on the problem addressed and the model proposed, which is twofold as follows: 1) the non-fragile state estimation problem is put forward for discrete time-delay neural networks in the presence of parameter uncertainties in all network parameters; and 2) the network output is quite general that is subject to nonlinear disturbances.

Notation: Throughout this paper, the superscript " $T$ " represents the matrix transposition. $\mathbb{R}$ denotes the set of real numbers; $\mathbb{R}^{n}$ denotes the $n$-dimensional Euclidean space; $\mathbb{R}^{n \times m}$ denotes the set of all $n \times m$ real matrices. $\mathbb{N}^{+}$stands for the sets of positive integers, $I$ and 0 denote the identity matrix and zero matrix of appropriate dimensions, respectively. We use $X>0(X<0)$ to denote a positive-definite matrix (negative-definite matrix) $X .|\cdot|$ is the Euclidean norm in $\mathbb{R}^{n}$. If $A$ is a matrix, $\lambda_{\min }$ stands for the smallest eigenvalue of $A$. The notation $*$ always denotes the symmetric block in a symmetric matrix, and $\operatorname{diag}\{\cdots\}$ stands for a block-diagonal matrix.

\section{PROBLEM FORMULATION}

In this paper, we consider a discrete-time neural network described by the following dynamical equations:

$$
\left\{\begin{array}{l}
x(k+1)=(C+\Delta C) x(k)+(A+\Delta A) f(x(k))+(B+\Delta B) f(x(k-d)) \\
x(k)=\phi(k), \quad k \in[-d, 0)
\end{array}\right.
$$

where $x(k)=\left[x_{1}(k), x_{2}(k), \cdots, x_{n}(k)\right]^{T} \in \mathbb{R}^{n}$ is the neural state vector; $f(x(k))=\left[f_{1}\left(x_{1}(k)\right), f_{2}\left(x_{2}(k)\right)\right.$, $\left.\cdots, f_{n}\left(x_{n}(k)\right)\right]^{T}$ represents the nonlinear activation function with the initial condition $f(0)=0 ; C=$ $\operatorname{diag}\left\{c_{1}, c_{2}, \cdots, c_{n}\right\}$ is a positive diagonal matrix; $A=\left[a_{i j}\right]_{n \times n}, B=\left[b_{i j}\right]_{n \times n}$ are, respectively, the connection weight matrix and the delayed connection weight matrix; $d \geq 0$ denotes the discrete timedelay; $\phi(k)$ describes the initial condition. In addition, $\Delta A, \Delta B$ and $\Delta C$ are time-varying parameter uncertainties that satisfy

$$
\left[\begin{array}{lll}
\Delta A & \Delta B & \Delta C
\end{array}\right]=M_{1} F_{1}(k)\left[\begin{array}{lll}
N_{1} & N_{2} & N_{3}
\end{array}\right]
$$

where $M_{1}, N_{1}, N_{2}, N_{3}$ are known real-valued matrices with appropriate dimensions and $F_{1}(k)$ is an unknown matrix satisfying

$$
F_{1}^{T}(k) F_{1}(k) \leq I, \quad \forall k \in \mathbb{N}^{+}
$$

Throughout this paper, we make the following assumption. 
Assumption 1: For any $\alpha_{1}, \alpha_{2} \in \mathbb{R}, \alpha_{1} \neq \alpha_{2}$, the activation function $f(\cdot)$ satisfies

$$
\gamma_{i}^{-} \leq \frac{f_{i}\left(\alpha_{1}\right)-f_{i}\left(\alpha_{2}\right)}{\alpha_{1}-\alpha_{2}} \leq \gamma_{i}^{+}, \quad(i=1,2, \cdots, n)
$$

where $\gamma_{i}^{-}$and $\gamma_{i}^{+}$are known constant scalars.

Remark 1: As shown in [22], the activation functions described in (1) are more general than the usual sigmoid functions and the commonly used Lipschitz conditions, where the constants $\gamma_{i}^{-}$and $\gamma_{i}^{+}$are allowed to be positive, negative or zero. Therefore, such activation functions could be nonmonotonic and would induce less conservative results.

The outputs from the neural network (1) are of the following form:

$$
y(k)=D x(k)+E g(k, x(k))
$$

where $y(k)=\left[y_{1}(k), y_{2}(k), \cdots, y_{n}(k)\right]^{T}$ represents the measurement output, $D$ and $E$ are known realvalued matrices with appropriate dimensions, and $g(k, x(k))$ is the neuron-dependent nonlinear disturbance that satisfies the following Lipschitz condition:

$$
\left|g\left(k, \mu_{1}\right)-g\left(k, \mu_{2}\right)\right| \leq\left|G\left(\mu_{1}-\mu_{2}\right)\right|
$$

where $G$ is a known constant matrix with appropriate dimension.

In order to estimate the state of the neural network (1) from available measurement output (5), we construct the following non-fragile state estimator:

$$
\left\{\begin{array}{l}
\hat{x}(k+1)=C \hat{x}(k)+A f(\hat{x}(k))+B f(\hat{x}(k-d))+(K+\Delta K)[y(k)-D \hat{x}(k)-E g(k, \hat{x}(k))] \\
\hat{x}(k)=\hat{\phi}(k), \quad k \in[-d, 0)
\end{array}\right.
$$

where $\hat{x}(k) \in \mathbb{R}^{n}$ is the state estimation, $\hat{\phi}(k)$ is the initial function of $\hat{x}(k)$, and $K$ is the estimator gain parameter to be determined. $\Delta K$ quantifies the estimator gain variation in the following additive norm-bounded form:

$$
\Delta K=M_{2} F_{2}(k) N_{4}
$$

where $M_{2}, N_{4}$ are known real-valued matrices with appropriate dimensions and $F_{2}(k)$ is an unknown matrix satisfying

$$
F_{2}^{T}(k) F_{2}(k) \leq I, \quad \forall k \in \mathbb{N}^{+}
$$

Letting the estimation error be $e(k)=x(k)-\hat{x}(k)$, the dynamics of the estimation error can be obtained from (1), (5) and (7) as follows:

$$
\begin{aligned}
e(k+1)= & (C-K D-\Delta K D) e(k)+\Delta C x(k)+A \tilde{f}(e(k))+\Delta A f(x(k))+B \tilde{f}(e(k-d)) \\
& +\Delta B f(x(k-d))-(K+\Delta K) E \tilde{g}(e(k))
\end{aligned}
$$

where

$$
\begin{aligned}
\tilde{f}(e(k)) & :=f(x(k))-f(\hat{x}(k)), \\
\tilde{f}(e(k-d)) & :=f(x(k-d))-f(\hat{x}(k-d), \\
\tilde{g}(e(k)) & :=g(k, x(k))-g(k, \hat{x}(k)) .
\end{aligned}
$$


From (4) and (6), we have that

$$
\begin{aligned}
& \gamma_{i}^{-} \leq \frac{\tilde{f}_{i}(e(k))}{e(k)} \leq \gamma_{i}^{+} \\
& |\tilde{g}(e(k))| \leq|G e(k)|
\end{aligned}
$$

Set

$$
\begin{aligned}
\eta(k) & =\left[\begin{array}{ll}
x^{T}(k) & e^{T}(k)
\end{array}\right]^{T}, \\
\varphi(\eta(k)) & =\left[\begin{array}{ll}
f^{T}(x(k)) & \tilde{f}^{T}(e(k))
\end{array}\right]^{T}, \\
\psi(\eta(k)) & =\left[\begin{array}{ll}
g^{T}(k, x(k)) & \tilde{g}^{T}(e(k))
\end{array}\right]^{T} .
\end{aligned}
$$

Considering (1) and (10), we obtain the following augmented system:

$$
\eta(k+1)=\tilde{C} \eta(k)+\tilde{A} \varphi(\eta(k))+\tilde{B} \varphi(\eta(k-d))-\tilde{E} \psi(\eta(k))
$$

where

$$
\begin{aligned}
& \tilde{C}=\bar{C}+\Delta \tilde{C}, \quad \tilde{A}=\bar{A}+\Delta \tilde{A}, \quad \tilde{B}=\bar{B}+\Delta \tilde{B}, \quad \tilde{E}=\bar{E}+\Delta \tilde{E}, \\
& \bar{C}=\operatorname{diag}\{C, C-K D\}, \quad \bar{A}=\operatorname{diag}\{A, A\}, \quad \bar{B}=\operatorname{diag}\{B, B\}, \quad \bar{E}=\operatorname{diag}\{0, K E\}, \\
& \Delta \tilde{C}=\left[\begin{array}{cc}
\Delta C & 0 \\
\Delta C & -\Delta K D
\end{array}\right], \quad \Delta \tilde{A}=\left[\begin{array}{cc}
\Delta A & 0 \\
\Delta A & 0
\end{array}\right], \quad \Delta \tilde{B}=\left[\begin{array}{cc}
\Delta B & 0 \\
\Delta B & 0
\end{array}\right], \quad \Delta \tilde{E}=\left[\begin{array}{cc}
0 & 0 \\
0 & \Delta K E
\end{array}\right] .
\end{aligned}
$$

Before proceeding further, we introduce the following definition.

Definition 1: The augmented system (13) is said to be asymptotically stable if, for any solution $\eta(k)$ of it, the following holds:

$$
\lim _{k \rightarrow \infty}|\eta(k)|^{2}=0
$$

The objective of this paper is to design appropriate estimator parameter $K$ for the state estimator (7) such that, in the presence of admissible gain variations as well as parameter uncertainties, the augmented system (13) is asymptotically stable, which implies that the error dynamics $e(k)$ tends to zero asymptotically.

\section{MAin Results}

In this section, the stability is analyzed for the augmented system (13). A sufficient condition is given to guarantee that the augmented system (13) is asymptotically stable and then the explicit expression of the desired estimator gain is proposed in terms of the solution to certain matrix inequality derived according to the obtained condition.

Before stating our main results, we introduce the following lemmas.

Lemma 1: [3] (Schur Complement) Given constant matrices $\mathcal{S}_{1}, \mathcal{S}_{2}, \mathcal{S}_{3}$ where $\mathcal{S}_{1}=\mathcal{S}_{1}^{\mathrm{T}}$ and $0<\mathcal{S}_{2}=$ $\mathcal{S}_{2}^{\mathrm{T}}$, then $\mathcal{S}_{1}+\mathcal{S}_{3}^{\mathrm{T}} \mathcal{S}_{2}^{-1} \mathcal{S}_{3}<0$ if and only if

$$
\left[\begin{array}{cc}
\mathcal{S}_{1} & \mathcal{S}_{3}^{\mathrm{T}} \\
\mathcal{S}_{3} & -\mathcal{S}_{2}
\end{array}\right]<0 \quad \text { or } \quad\left[\begin{array}{cc}
-\mathcal{S}_{2} & \mathcal{S}_{3} \\
\mathcal{S}_{3}^{\mathrm{T}} & \mathcal{S}_{1}
\end{array}\right]<0 .
$$


Lemma 2: [3] (S-procedure) Let $\mathcal{U}, \mathcal{V}(t), \mathcal{W}$ and $\mathcal{Q}$ be real matrices of appropriate dimensions with $\mathcal{Q}$ satisfying $\mathcal{Q}=\mathcal{Q}^{T}$. Then, for all $\mathcal{V}(t) \mathcal{V}^{T}(t) \leq I$,

$$
\mathcal{Q}+\mathcal{U} \mathcal{V}(t) \mathcal{W}+\mathcal{W}^{T} \mathcal{V}^{T}(t) \mathcal{U}^{T}<0
$$

holds if and only if there exists a positive scalar $\mu$ such that

$$
\mathcal{Q}+\mu^{-1} \mathcal{U} \mathcal{U}^{T}+\mu \mathcal{W}^{T} \mathcal{W}<0
$$

Lemma 3: [22] Suppose that $\mathcal{B}=\operatorname{diag}\left\{\beta_{1}, \beta_{2}, \cdots, \beta_{n}\right\}$ is a positive-semidefinite diagonal matrix. Let $x=\left[x_{1}, x_{2}, \cdots, x_{n}\right]^{T} \in \mathbb{R}^{n}$ and $\mathcal{H}(x)=\left[h_{1}\left(x_{1}\right), h_{2}\left(x_{2}\right), \cdots, h_{n}\left(x_{n}\right)\right]^{T}$ be a continuous nonlinear function satisfying

$$
l_{i}^{-} \leq \frac{h_{i}(m)}{m} \leq l_{i}^{+}, \quad m \neq 0, \quad m \in \mathbb{R}, \quad i=1,2, \cdots, n
$$

with $l_{i}^{-}$and $l_{i}^{+}$being constant scalars. Then

$$
\left[\begin{array}{c}
x \\
\mathcal{H}(x)
\end{array}\right]^{T}\left[\begin{array}{cc}
\mathcal{B} L_{1} & -\mathcal{B} L_{2} \\
-\mathcal{B} L_{2} & \mathcal{B}
\end{array}\right]\left[\begin{array}{c}
x \\
\mathcal{H}(x)
\end{array}\right] \leq 0
$$

or

$$
x^{T} \mathcal{B} L_{1} x-2 x^{T} \mathcal{B} L_{2} \mathcal{H}(x)+\mathcal{H}^{T}(x) \mathcal{B H}(x) \leq 0
$$

where $L_{1}=\operatorname{diag}\left\{l_{1}^{+} l_{1}^{-}, l_{2}^{+} l_{2}^{-}, \cdots, l_{n}^{+} l_{n}^{-}\right\}$and $L_{2}=\operatorname{diag}\left\{\frac{l_{1}^{+}+l_{1}^{-}}{2}, \frac{l_{2}^{+}+l_{2}^{-}}{2}, \cdots, \frac{l_{n}^{+}+l_{n}^{-}}{2}\right\}$.

Let us now consider the stability analysis problem for the augmented system (13).

Theorem 1: Consider the neural network model (1) and suppose that the estimator parameter $K$ is given. The augmented system (13) is asymptotically stable if there exist a positive constant $\varepsilon$, symmetric positive definite matrices $P>0, Q>0, R>0$ and four sets of diagonal matrices $F>0, H>0, X>0$, $Y>0$ satisfying the following inequality

$$
\tilde{\Gamma}=\left[\begin{array}{ccccc}
\Omega_{1} & * & * & * & * \\
0 & \Omega_{2} & * & * & * \\
\Omega_{3} & 0 & \Omega_{4} & * & * \\
\tilde{B}^{T} P \tilde{C} & -\Lambda_{22} & \tilde{B}^{T} P \tilde{A} & \Omega_{5} & * \\
-\tilde{E}^{T} P \tilde{C} & 0 & -\tilde{E}^{T} P \tilde{A} & -\tilde{E}^{T} P \tilde{B} & \Omega_{6}
\end{array}\right]<0
$$

where

$$
\begin{aligned}
& \bar{I}=\left[\begin{array}{ll}
0 & I
\end{array}\right], \quad \bar{G}=\left[\begin{array}{ll}
0 & G
\end{array}\right], \quad \Lambda_{11}=\left[\begin{array}{cc}
F \Lambda_{1} & 0 \\
0 & X \Lambda_{1}
\end{array}\right], \quad \Lambda_{21}=\left[\begin{array}{cc}
-F \Lambda_{2} & 0 \\
0 & -X \Lambda_{2}
\end{array}\right], \\
& \Lambda_{12}=\left[\begin{array}{cc}
H \Lambda_{1} & 0 \\
0 & Y \Lambda_{1}
\end{array}\right], \quad \Lambda_{22}=\left[\begin{array}{cc}
-H \Lambda_{2} & 0 \\
0 & -Y \Lambda_{2}
\end{array}\right], \quad \bar{F}=\left[\begin{array}{cc}
F & 0 \\
0 & X
\end{array}\right], \quad \bar{H}=\left[\begin{array}{cc}
H & 0 \\
0 & Y
\end{array}\right], \\
& \Gamma_{1}=\tilde{C}^{T} P \tilde{C}-P+Q, \quad \Gamma_{2}=\tilde{A}^{T} P \tilde{A}+R, \quad \Gamma_{3}=\tilde{B}^{T} P \tilde{B}-R, \\
& \Omega_{1}=\Gamma_{1}-\Lambda_{11}+\varepsilon \bar{G}^{T} \bar{G}, \quad \Omega_{2}=-Q-\Lambda_{12}, \quad \Omega_{3}=\tilde{A}^{T} P \tilde{C}-\Lambda_{21}, \\
& \Omega_{4}=\Gamma_{2}-\bar{F}, \quad \Omega_{5}=\Gamma_{3}-\bar{H}, \quad \Omega_{6}=\tilde{E}^{T} P \tilde{E}-\varepsilon \bar{I}^{T} \bar{I},
\end{aligned}
$$




$$
\Lambda_{1}=\operatorname{diag}\left\{\gamma_{1}^{+} \gamma_{1}^{-}, \gamma_{2}^{+} \gamma_{2}^{-}, \cdots, \gamma_{n}^{+} \gamma_{n}^{-}\right\}, \quad \Lambda_{2}=\operatorname{diag}\left\{\frac{\gamma_{1}^{+}+\gamma_{1}^{-}}{2}, \frac{\gamma_{2}^{+}+\gamma_{2}^{-}}{2}, \cdots, \frac{\gamma_{n}^{+}+\gamma_{n}^{-}}{2}\right\}
$$

Proof: Choose the following Lyapunov-Krasovskii functional for system (13):

$$
V(x(k), k)=\eta^{T}(k) P \eta(k)+\sum_{i=k-d}^{k-1} \eta^{T}(i) Q \eta(i)+\sum_{i=k-d}^{k-1} \varphi^{T}(\eta(i)) R \varphi^{T}(\eta(i)) .
$$

For $P>0, Q>0$ and $R>0$, we have

$$
\begin{aligned}
\Delta V(k)= & V(x(k+1), k+1)-V(x(k), k) \\
= & \eta^{T}(k+1) P \eta(k+1)+\sum_{i=k-d+1}^{k} \eta^{T}(i) Q \eta(i)+\sum_{i=k-d+1}^{k} \varphi^{T}(\eta(i)) R \varphi(\eta(i)) \\
& -\eta^{T}(k) P \eta(k)-\sum_{i=k-d}^{k-1} \eta^{T}(i) Q \eta(i)-\sum_{i=k-d}^{k-1} \varphi^{T}(\eta(i)) R \varphi(\eta(i)) \\
= & \left.\eta^{T}(k) \tilde{C}^{T}+\varphi^{T}(\eta(k)) \tilde{A}^{T}+\varphi^{T}(\eta(k-d)) \tilde{B}^{T}-\psi^{T}(\eta(k)) \tilde{E}^{T}\right] P[\tilde{C} \eta(k) \\
& +\tilde{A} \varphi(\eta(k))+\tilde{B} \varphi(\eta(k-d))-\tilde{E} \psi(\eta(k))]-\eta^{T}(k) P \eta(k)+\eta^{T}(k) Q \eta(k) \\
& -\eta^{T}(k-d) Q \eta(k-d)+\varphi^{T}(\eta(k)) R \varphi(\eta(k))-\varphi^{T}(\eta(k-d)) R \varphi(\eta(k-d)) \\
= & \xi^{T}(k) \Gamma \xi(k)
\end{aligned}
$$

where

$$
\begin{aligned}
& \xi(k):=\left[\begin{array}{lllll}
\eta^{T}(k) & \eta^{T}(k-d) & \varphi^{T}(\eta(k)) & \varphi^{T}(\eta(k-d)) & \psi^{T}(\eta(k))
\end{array}\right]^{T}, \\
& \Gamma=\left[\begin{array}{ccccc}
\Gamma_{1} & * & * & * & * \\
0 & -Q & * & * & * \\
\tilde{A}^{T} P \tilde{C} & 0 & \Gamma_{2} & * & * \\
\tilde{B}^{T} P \tilde{C} & 0 & \tilde{B}^{T} P \tilde{A} & \Gamma_{3} & * \\
-\tilde{E}^{T} P \tilde{C} & 0 & -\tilde{E}^{T} P \tilde{A} & -\tilde{E}^{T} P \tilde{B} & \tilde{E}^{T} P \tilde{E}
\end{array}\right]
\end{aligned}
$$

Moreover, it follows from Assumption 1 and Lemma 3 that

$$
\begin{gathered}
{\left[\begin{array}{c}
x(k) \\
f(x(k))
\end{array}\right]^{T}\left[\begin{array}{cc}
F \Lambda_{1} & -F \Lambda_{2} \\
-F \Lambda_{2} & F
\end{array}\right]\left[\begin{array}{c}
x(k) \\
f(x(k))
\end{array}\right] \leq 0} \\
{\left[\begin{array}{c}
e(k) \\
\tilde{f}(e(k))
\end{array}\right]^{T}\left[\begin{array}{cc}
X \Lambda_{1} & -X \Lambda_{2} \\
-X \Lambda_{2} & X
\end{array}\right]\left[\begin{array}{c}
e(k) \\
\tilde{f}(e(k))
\end{array}\right] \leq 0} \\
{\left[\begin{array}{c}
x(k-d) \\
f(x(k-d))
\end{array}\right]^{T}\left[\begin{array}{cc}
H \Lambda_{1} & -H \Lambda_{2} \\
-H \Lambda_{2} & H
\end{array}\right]\left[\begin{array}{c}
x(k-d) \\
f(x(k-d))
\end{array}\right] \leq 0} \\
{\left[\begin{array}{c}
e(k-d) \\
\tilde{f}(e(k-d))
\end{array}\right]^{T}\left[\begin{array}{cc}
Y \Lambda_{1} & -Y \Lambda_{2} \\
-Y \Lambda_{2} & Y
\end{array}\right]\left[\begin{array}{c}
e(k-d) \\
\tilde{f}(e(k-d))
\end{array}\right] \leq 0}
\end{gathered}
$$

From (15)-(18), we have immediately that

$$
\left[\begin{array}{c}
\eta(k) \\
\varphi(\eta(k))
\end{array}\right]^{T}\left[\begin{array}{cc}
\Lambda_{11} & \Lambda_{21} \\
\Lambda_{21} & \bar{F}
\end{array}\right]\left[\begin{array}{c}
\eta(k) \\
\varphi(\eta(k))
\end{array}\right] \leq 0
$$




$$
\left[\begin{array}{c}
\eta(k-d) \\
\varphi(\eta(k-d))
\end{array}\right]^{T}\left[\begin{array}{cc}
\Lambda_{12} & \Lambda_{22} \\
\Lambda_{22} & \bar{H}
\end{array}\right]\left[\begin{array}{c}
\eta(k-d) \\
\varphi(\eta(k-d))
\end{array}\right] \leq 0
$$

Furthermore, it follows from (6) that

$$
\begin{aligned}
\psi^{T}(\eta(k)) \bar{I}^{T} \bar{I} \psi(\eta(k)) & =[\bar{I} \psi(\eta(k))]^{T}[\bar{I} \psi(\eta(k))]=\tilde{g}^{T}(e(k)) \tilde{g}(e(k)) \\
& =|g(x(k))-g(\hat{x}(k))|^{2} \leq|G e(k)|^{2}=e^{T}(k) G^{T} G e(k) \\
& =\eta^{T}(k) \bar{G}^{T} \bar{G} \eta(k) .
\end{aligned}
$$

Then, for a positive scalar $\varepsilon$, one can obtain

$$
0 \leq \varepsilon\left[\eta^{T}(k) \bar{G}^{T} \bar{G} \eta(k)-\psi^{T}(\eta(k)) \bar{I}^{T} \bar{I} \psi(\eta(k))\right]
$$

From (19)-(20), it is not difficult to derive that

$$
\begin{aligned}
\Delta V(k)=\xi^{T}(k) \Gamma \xi(k) \leq & \xi^{T}(k) \Gamma \xi(k)-\left[\begin{array}{c}
\eta(k) \\
\varphi(\eta(k))
\end{array}\right]^{T}\left[\begin{array}{cc}
\Lambda_{11} & \Lambda_{21} \\
\Lambda_{21} & \bar{F}
\end{array}\right]\left[\begin{array}{c}
\eta(k) \\
\varphi(\eta(k))
\end{array}\right] \\
& -\left[\begin{array}{c}
\eta(k-d) \\
\varphi(\eta(k-d))
\end{array}\right]^{T}\left[\begin{array}{cc}
\Lambda_{12} & \Lambda_{22} \\
\Lambda_{22} & \bar{H}
\end{array}\right]\left[\begin{array}{c}
\eta(k-d) \\
\varphi(\eta(k-d))
\end{array}\right] \\
& +\varepsilon\left[\eta^{T}(k) \bar{G}^{T} \bar{G} \eta(k)-\psi^{T}(\eta(k)) \bar{I}^{T} \bar{I} \psi(\eta(k))\right] \\
= & \xi^{T}(k) \tilde{\Gamma} \xi(k)
\end{aligned}
$$

In terms of inequality (14), we have

$$
\Delta V(k) \leq-\lambda_{\min }(-\tilde{\Gamma})|\xi(k)|^{2}<0
$$

which implies

$$
\Delta V(k) \leq-\lambda_{\min }(-\tilde{\Gamma})|\xi(k)|^{2}
$$

Given a positive integer $m$, the recursive sum of both sides of (23) from 0 to $m$ leads to

$$
V(x(m+1), m+1)-V(x(0), 0) \leq-\lambda_{\min }(-\tilde{\Gamma}) \sum_{k=0}^{m}|\xi(k)|^{2}
$$

which results in

$$
\sum_{k=0}^{m}|\xi(k)|^{2} \leq \frac{1}{\lambda_{\min }(-\tilde{\Gamma})} V(x(0), 0)
$$

Letting $m \rightarrow \infty$, we know that the series $\sum_{k=0}^{m}|\xi(k)|^{2}$ is convergent, which means

$$
\lim _{k \rightarrow \infty}|\eta(k)|^{2}=0
$$

The proof is now complete.

Having conducted the estimating performance analysis in Theorem 1, we are now in a position to deal with the problem of designing estimator and the main results are given in the following theorem.

Theorem 2: There exists a non-fragile state estimator of the type (7) with $\Delta K$ satisfying (8) such that the augmented system (14) is asymptotically stable if there exist positive constants $\varepsilon$ and $\epsilon$, symmetric positive definite matrices $P=\operatorname{diag}\left\{P_{1}, P_{2}\right\}>0, Q>0$ and $R>0$, four sets of diagonal matrices $F>0$, 
$H>0, X>0, Y>0$ and a matrix $Z$ with appropriate dimensions such that the following linear matrix inequality (LMI)

$$
\left[\begin{array}{ccccccc}
\Theta_{1} & * & * & * & * & * & * \\
0 & \Omega_{2} & * & * & * & * & * \\
-\Lambda_{21} & 0 & \Theta_{2} & * & * & * & * \\
0 & -\Lambda_{22} & 0 & \Theta_{3} & * & * & * \\
0 & 0 & 0 & 0 & \Theta_{4} & * & * \\
\Theta_{5} & 0 & P \bar{A} & P \bar{B} & -Z_{2} & -P & * \\
0 & 0 & 0 & 0 & 0 & \mathcal{D}_{1}^{T} & -\epsilon I
\end{array}\right]<0
$$

holds, where

$$
\begin{aligned}
& \tilde{M}_{1}=\left[\begin{array}{cc}
M_{1} & 0 \\
M_{1} & M_{2}
\end{array}\right], \quad \bar{M}_{1}=\left[\begin{array}{cc}
M_{1} & 0 \\
M_{1} & 0
\end{array}\right], \quad \bar{M}_{2}=\left[\begin{array}{cc}
0 & 0 \\
0 & M_{2}
\end{array}\right], \\
& Z_{1}=\left[\begin{array}{cc}
0 & 0 \\
0 & Z D
\end{array}\right], \quad Z_{2}=\left[\begin{array}{cc}
0 & 0 \\
0 & Z E
\end{array}\right], \quad \hat{C}=\operatorname{diag}\{C, C\}, \\
& \bar{N}_{1}=\operatorname{diag}\left\{N_{1}, N_{1}\right\}, \quad \bar{N}_{2}=\operatorname{diag}\left\{N_{2}, N_{2}\right\}, \quad \bar{N}_{3}=\operatorname{diag}\left\{N_{3},-N_{4} D\right\}, \quad \bar{N}_{4}=\operatorname{diag}\left\{N_{4}, N_{4} E\right\}, \\
& \mathcal{D}_{1}=\left[\begin{array}{llllll}
P \tilde{M}_{1} & 0 & P \bar{M}_{1} & P \bar{M}_{1} & P \bar{M}_{2} & 0
\end{array}\right], \quad \Theta_{1}=-P+Q-\Lambda_{11}+\varepsilon \bar{G}^{T} \bar{G}+\epsilon \bar{N}_{3}^{T} \bar{N}_{3}, \\
& \Theta_{2}=R-\bar{F}+\epsilon \bar{N}_{1}^{T} \bar{N}_{1}, \Theta_{3}=-R-\bar{H}+\epsilon \bar{N}_{2}^{T} \bar{N}_{2}, \Theta_{4}=-\varepsilon \bar{I}^{T} \bar{I}+\epsilon \bar{N}_{4}^{T} \bar{N}_{4}, \Theta_{5}=P \hat{C}-Z_{1} \text {. }
\end{aligned}
$$

and other parameters are defined as those in Theorem 1. In this case, the estimator gain $K$ of the desired non-fragile state estimator (7) can be characterized by

$$
K=P_{2}^{-1} Z
$$

Proof: First, we denote

$$
\begin{aligned}
& \Xi_{1}=-P+Q-\Lambda_{11}+\varepsilon \bar{G}^{T} \bar{G}, \quad \Xi_{2}=R-\bar{F}, \quad \Xi_{3}=-R-\bar{H} \\
& \Sigma_{1}=\left[\begin{array}{ccccc}
\Xi_{1} & * & * & * & * \\
0 & \Omega_{2} & * & * & * \\
-\Lambda_{21} & 0 & \Xi_{2} & * & * \\
0 & -\Lambda_{22} & 0 & \Xi_{3} & * \\
0 & 0 & 0 & 0 & -\varepsilon \bar{I}^{T} \bar{I}
\end{array}\right], \quad \Sigma_{2}=\left[\begin{array}{ccccc}
P \tilde{C} & 0 & P \tilde{A} & P \tilde{B} & -P \tilde{E}
\end{array}\right]
\end{aligned}
$$

and then $\tilde{\Gamma}$ in (14) can be rewritten as

$$
\tilde{\Gamma}=\Sigma_{1}+\Sigma_{2}^{T} P^{-1} \Sigma_{2} \leq 0
$$

which, according to Lemma 1 , is equivalent to

$$
\left[\begin{array}{cc}
\Sigma_{1} & \Sigma_{2}^{\mathrm{T}} \\
\Sigma_{2} & -P
\end{array}\right]<0
$$


Replacing $\tilde{C}, \tilde{A}, \tilde{B}$ and $\tilde{E}$ with $\bar{C}+\Delta \tilde{C}, \bar{A}+\Delta \tilde{A}, \bar{B}+\Delta \tilde{B}$ and $\bar{E}+\Delta \tilde{E}$ in (28), respectively, one obtains

$$
\left[\begin{array}{cccccc}
\Xi_{1} & * & * & * & * & * \\
0 & \Omega_{2} & * & * & * & * \\
-\Lambda_{21} & 0 & \Xi_{2} & * & * & * \\
0 & -\Lambda_{22} & 0 & \Xi_{3} & * & * \\
0 & 0 & 0 & 0 & -\varepsilon \bar{I}^{T} \bar{I} & * \\
P \bar{C} & 0 & P \bar{A} & P \bar{B} & -P \bar{E} & -P
\end{array}\right]+\mathcal{D} \mathcal{F}(k) \mathcal{N}+\mathcal{N}^{T} \mathcal{F}^{T}(k) \mathcal{D}^{T} \leq 0
$$

where

$\mathcal{D}=\left[\begin{array}{llllll}0 & 0 & 0 & 0 & 0 & \mathcal{D}_{1}^{T}\end{array}\right]^{T}, \quad \mathcal{N}=\operatorname{diag}\left\{\begin{array}{llllll}\bar{N}_{3} & 0 & \bar{N}_{1} & \bar{N}_{2} & -\bar{N}_{4} & 0\end{array}\right\}$,

$\mathcal{F}(k)=\operatorname{diag}\left\{\operatorname{diag}\left\{F_{1}(k), F_{2}(k)\right\}, 0, \operatorname{diag}\left\{F_{1}(k), F_{1}(k)\right\}, \operatorname{diag}\left\{F_{1}(k), F_{1}(k)\right\}, \operatorname{diag}\left\{F_{2}(k), F_{2}(k)\right\}, 0\right\}$.

Employing Lemmas 1-2 again and applying the change of variable such that $K=P_{2}^{-1} Z$, we can see that $\tilde{\Gamma}=\Sigma_{1}+\Sigma_{2}^{T} P^{-1} \Sigma_{2} \leq 0$ is guaranteed by the LMI (26). Therefore, the system (13) is globally asymptotically stable and the proof is then completed.

Remark 2: It is worth noting that the non-fragile state estimation problem is introduced for the discretetime RNNs with time-delays because the implementation of the designed controller/estimator is often inaccurate due to a variety of reasons such as analog-digital and digital-analog conversion, finite word length, finite resolution measuring instruments, programming errors, and roundoff errors in numerical computations. In Theorems 1-2, sufficient conditions for the existence and the derivation of the desired state estimator are provided, respectively. It is observed that all the network parameters, the sector-bounds of the activation function, the bounds of the Lipschitz-type nonlinearities in the network output as well as the bounds of the parameter uncertainties are all reflected in the main results. The obtained state estimator is capable of tolerating the admissible gain variations that might occur in the physical implementation. Conditions in Theorem 2 can be readily solved by utilizing the well-developed interior-point methods embedded in the Matlab software.

\section{An ILlustrative EXAMPle}

In this section, we present a simulation example to illustrate the effectiveness of the developed theoretical results. 
Consider an uncertain delayed neural network (1) with the following parameters:

$$
\begin{aligned}
& C=\left[\begin{array}{ccc}
0.2 & 0 & 0 \\
0 & 0.3 & 0 \\
0 & 0 & 0.15
\end{array}\right], A=\left[\begin{array}{ccc}
-0.1 & 0.6 & 0.1 \\
0.7 & 0.2 & 0.2 \\
0.1 & -0.2 & 0.3
\end{array}\right], B=\left[\begin{array}{ccc}
0.2 & -0.2 & 0.1 \\
0.1 & 0.2 & 0.1 \\
0.4 & -0.1 & 0.3
\end{array}\right], M_{1}=\left[\begin{array}{c}
0.3 \\
0.2 \\
-0.1
\end{array}\right], \\
& M_{2}=\left[\begin{array}{ccc}
0.2 & 0 & 0 \\
0 & 0.2 & 0 \\
0 & 0 & 0.2
\end{array}\right], N_{1}=\left[\begin{array}{lll}
0.1 & 0.2 & 0.1
\end{array}\right], N_{2}=\left[\begin{array}{lll}
0.1 & -0.2 & 0.1
\end{array}\right] \text {, } \\
& N_{3}=\left[\begin{array}{lll}
0.1 & 0.2 & -0.1
\end{array}\right], N_{4}=\left[\begin{array}{ccc}
0.3 & 0 & 0 \\
0 & 0.3 & 0 \\
0 & 0 & 0.3
\end{array}\right], d=5, F_{1}(k)=\sin (0.6 k), F_{2}(k)=\sin (0.6 k) \text {. }
\end{aligned}
$$

The parameters of the network measurement (5) are given as

$$
D=I, \quad E=1, \quad g(k, x(k))=0.4 \cos (x(k)), \quad G=0.4 I .
$$

Take the activation functions as follows:

$$
f_{1}(s)=-\tanh (0.4 s), \quad f_{2}(s)=0.2 \tanh (s), \quad f_{3}(s)=\tanh (0.6 s) .
$$

It can be calculated that $\Lambda_{1}=0, \Lambda_{2}=\operatorname{diag}\{0.2,0.1,0.3\}$. By solving the LMI (26), the state estimator gain matrix can be obtained as follows:

$$
K=\left[\begin{array}{ccc}
0.1775 & 0.1924 & 0.0065 \\
0.1034 & 0.3321 & -0.0152 \\
0.0173 & -0.0427 & 0.1831
\end{array}\right]
$$

The simulation results are shown in Figs. 1-4, where the true states $x_{1}(k), x_{2}(k), x_{3}(k)$ and their estimates $\hat{x}_{1}(k), \hat{x}_{2}(k), \hat{x}_{3}(k)$ are depicted, respectively, in Fig. 1, Fig. 2 and Fig. 3 with the initial condition

$$
x(0)=\left[\begin{array}{lll}
-0.12 & 0.28 & -0.52
\end{array}\right]^{T}, \quad \hat{x}(0)=\left[\begin{array}{lll}
0.67 & -1.14 & 0.2
\end{array}\right]^{T} .
$$

Fig. 4 shows the dynamical evolution of the estimate error $e_{1}(k), e_{2}(k), e_{3}(k)$. The simulation results verify the effectiveness of the developed algorithm for designing the non-fragile state estimator for discrete-time neural networks with parameter uncertainties.

\section{CONCLUSIONS}

In this paper, the non-fragile state estimation problem has been studied for a class of uncertain discretetime neural networks with time-delay. By employing the Lyapunov stability theory and the matrix analysis technique, a sufficient condition has been established to ensure that the dynamics of the estimation error achieves the asymptotic stability for all admissible parameter uncertainties as well as gain variations. The explicit expression of the gain matrix of the desired non-fragile estimator has been characterized by means of the feasibility to a linear matrix inequality. Finally, an example has been given to illustrate the 


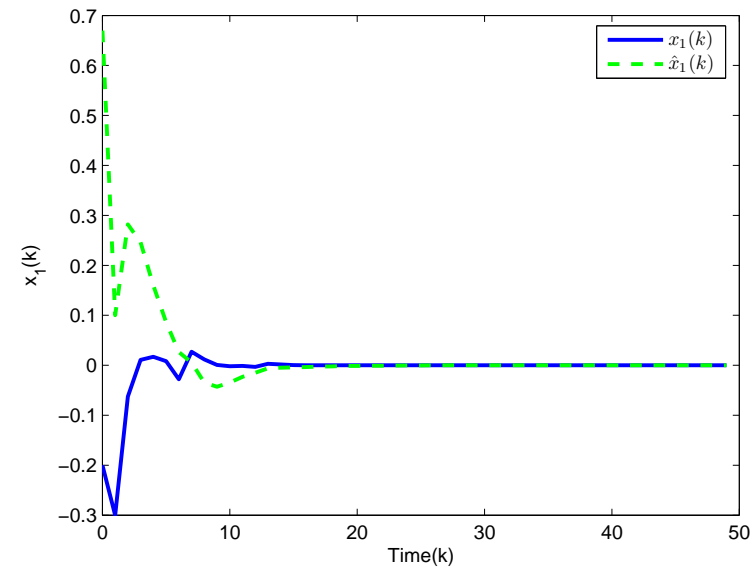

Fig. 1: The true states of $x_{1}(k)$ and its estimate.

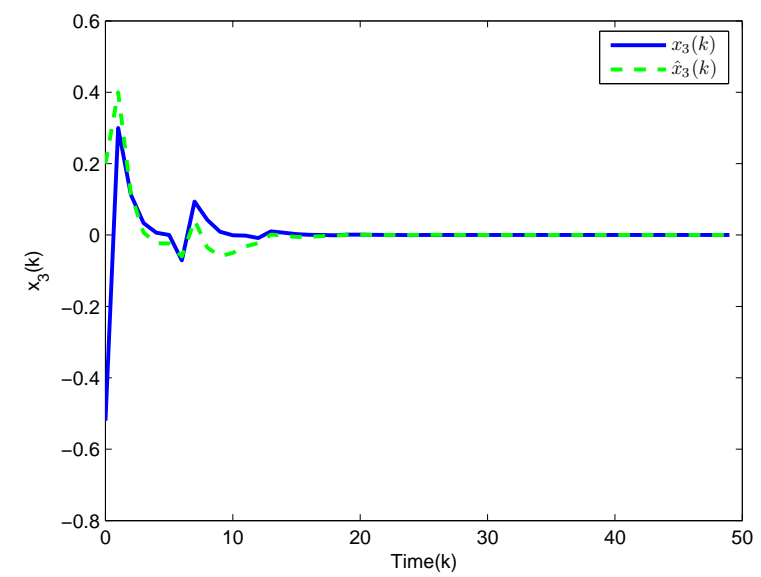

Fig. 3: The true states of $x_{3}(k)$ and its estimate.

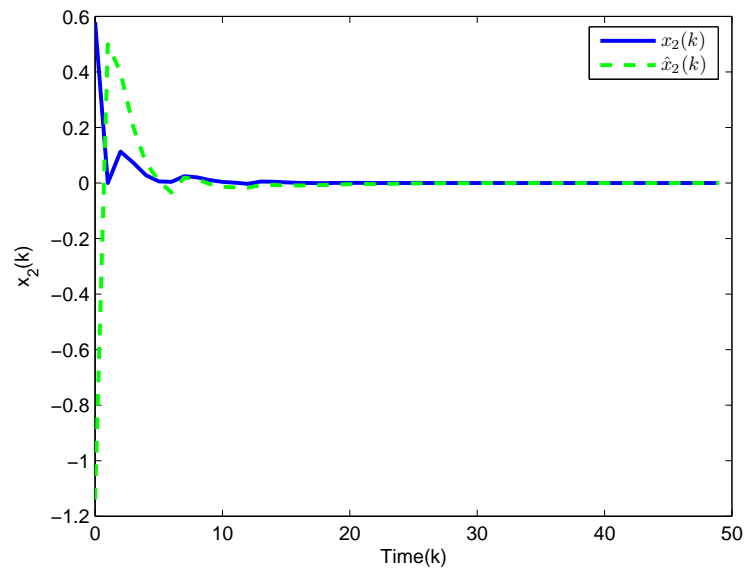

Fig. 2: The true states of $x_{2}(k)$ and its estimate.

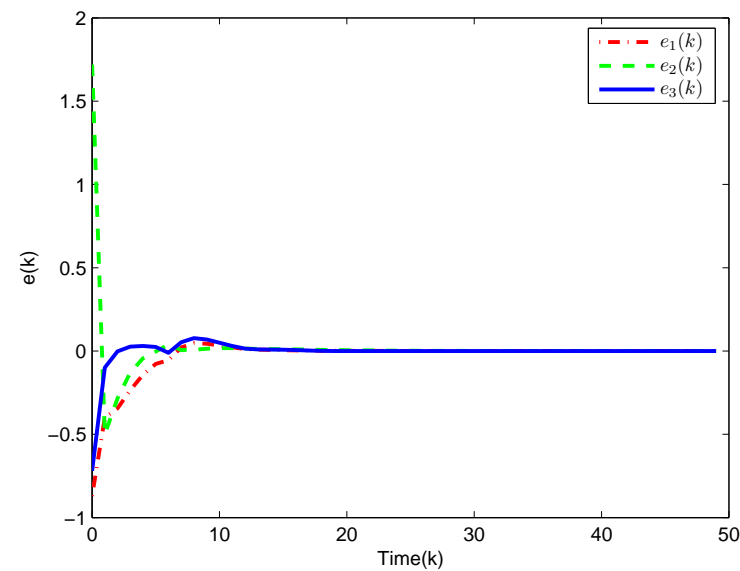

Fig. 4: The estimation errors.

usefulness of the developed state estimation approach. The results in this paper could be further extended to the non-fragile state estimation problems for discrete neural networks with more complicated networkinduced phenomena such as fading measurements [4], [5], [10], [20], [26], missing measurements [8], sensor delays [9], randomly occurring faults [14] and mixed time-delays [31].

\section{REFERENCES}

[1] S. Arik, An analysis of exponential stability of delayed neural networks with time varying delays, Neural Networks, Vol. 17, pp. 10271031, 2004.

[2] P. Baldi and A. F. Atiya, How delays affect neural dynamics and learning, IEEE Transactions on Neural Networks, Vol. 5, No. 4, pp. 612-621, 1994.

[3] S. Boyd, L. E. Ghaoui, E. Feron and V. Balakrishnan, Linear Matrix Inequalities in System and Control Theory, Society for Industry and Applied Mathematics, Philadelphia, 1994. 
[4] D. Ding, Z. Wang, J. Lam and B. Shen, Finite-Horizon $H_{\infty}$ control for discrete time-varying systems with randomly occurring nonlinearities and fading measurements, IEEE Transactions on Automatic Control, Vol. 60, No. 9, pp. 2488-2493, 2015.

[5] D. Ding, Z. Wang, B. Shen and H. Dong, Envelope-constrained $H_{\infty}$ filtering with fading measurements and randomly occurring nonlinearities: the finite horizon case, Automatica, Vol. 55, May 2015, pp. 37-45.

[6] T. Ensari and S. Arik, Global stability of neural networks with multiple time varying delays, IEEE Transactions on Automatica Control, Vol. 50, No. 11, pp. 1781-1785, 2005.

[7] H. Dong, Z. Wang, S. X. Ding and H. Gao, Finite-horizon reliable control with randomly occurring uncertainties and nonlinearities subject to output quantization, Automatica, Vol. 52, pp. 355-362, 2015.

[8] J. Hu, Z. Wang, B. Shen and H. Gao, Quantized recursive filtering for a class of nonlinear systems with multiplicative noises and missing measurements, International Journal of Control, Vol. 86, No. 4, pp. 650-663, 2013.

[9] J. Hu, Z. Wang, B. Shen and H. Gao, Gain-constrained recursive filtering with stochastic nonlinearities and probabilistic sensor delays, IEEE Transactions on Signal Processing, Vol. 61, No. 5, pp. 1230-1238, 2013.

[10] H. Dong, Z. Wang, S. X. Ding and H. Gao, Event-based $H_{\infty}$ filter design for a class of nonlinear time-varying systems with fading channels and multiplicative noises, IEEE Transactions on Signal Processing, Vol. 63, No. 13, pp. 3387-3395, 2015.

[11] C. Feng and R. Plamondon, On the stability analysis of delayed neural networks systems, Neural Networks, Vol. 14, No. 9, pp. 11811188, 2001.

[12] H. J. Gao and C. H. Wang, A delay-dependent approach to robust $H_{\infty}$ filtering for uncertain discrete-time state-delayed systems, IEEE Transactions Signal Process, Vol. 52, No. 6, pp. 1631-1640, 2004.

[13] Y. He, G. P. Liu, D. Rees and M. Wu, Stability analysis for neural networks with time-varying interval delay, IEEE Transactions on Neural Networks, Vol. 18, No. 6, pp. 1850-1854, 2007.

[14] H. Dong, Z. Wang, S. X. Ding and H. Gao, Finite-horizon estimation of randomly occurring faults for a class of nonlinear time-varying systems, Automatica, Vol. 50, No. 12, pp. 3182-3189, 2014.

[15] Y. He, Q. G. Wang, M. Wu and C. Lin, Delay-dependent state estimation for delayed neural networks, IEEE Transactions on Neural Networks, Vol. 17, No. 4, pp. 1077-1081, 2006.

[16] J. Hu, D. Chen and J. Du, State estimation for a class of discrete nonlinear systems with randomly occurring uncertainties and distributed sensor delays, International Journal of General Systems, Vol. 43, No. 3-4, pp. 387-401, 2014.

[17] H. Huang, G. Feng and J. Cao, Robust state estimation for uncertain neural networks with time-varying delay, IEEE Transactions on Neural Networks, Vol. 19, No. 8, pp. 1329-1339, 2008.

[18] J. Jian and Z. Zhao, Global stability in Lagrange sense for BAM-type Cohen-Grossberg neural networks with time-varying delays, Systems Science and Control Engineering: An Open Access Journal, Vol. 3, No. 1, pp. 1-7, 2015.

[19] L. Keel and S. Bhattacharyya, Robust, fragile, or optimal, IEEE Transactions on Automatic Control, Vol. 42, pp.1098-1105, 1997.

[20] D. Ding, Z. Wang, B. Shen and H. Dong, $H_{\infty}$ state estimation with fading measurements, randomly varying nonlinearities and probabilistic distributed delays, International Journal of Robust and Nonlinear Control, Vol. 25, No. 13, pp. $2180-2195,2015$.

[21] J. Lian and J. Wang, Passivity of switched recurrent neural networks with time-varying delays, IEEE Transactions on Neural Networks and Learning Systems, Vol. 26, No. 2, pp. 357-366, 2015.

[22] Y. Liu, Z. Wang and X. Liu, Global exponential stability of generalized recurrent neural networks with discrete and distributed delays, Neural Networks, Vol. 19, No. 5, pp. 667-675, 2006.

[23] X. Lou and B. Cui, Design of state estimator for uncertain neural networks via the integral-inequality method, Nonlinear Dynamics, Vol. 53, pp. 223-235, 2008.

[24] Y. Ma, N. Gu and Q. Zhang, Non-fragile robust $H_{\infty}$ control for uncertain discrete-time singular systems with time-varying delays, Journal of the Franklin Institute, Vol. 351, No. 6, pp. 3163-3181, 2014.

[25] S. Mou, H. Gao, W. Qiang and Z. Fei, State estimation for discrete-time neural networks with time-varying delays, Neurocomputing, Vol. 72, No. 1-3, pp. 643-647, 2008.

[26] H. Dong, Z. Wang, S. X. Ding and H. Gao, On $H_{\infty}$ estimation of randomly occurring faults for a class of nonlinear time-varying systems with fading channels, IEEE Transactions on Automatic Control, accepted for publication, DOI: 10.1109/TAC.2015.2437526.

[27] J. H. Park, Robust non-fragile control for uncertain discrete-delay large-scale systems with a class of controller gain variations, Applied Mathematics and Computation, Vol. 149, pp. 147-164, 2004.

[28] R. Samli and E. Yucel, Global robust stability analysis of uncertain neural networks with time varying delays, Neurocomputing, Vol. 167, pp. 371-377, 2015. 
[29] S. Senan, Robustness analysis of uncertain dynamical neural networks with multiple time delays, Neural Networks, Vol. 70, pp. 53-60, 2015.

[30] B. Shen, Z. Wang, D. Ding and H. Shu, $H_{\infty}$ state estimation for complex networks with uncertain inner coupling and incomplete measurements, IEEE Transactions on Neural Networks and Learning Systems, Vol. 24, No. 12, pp. 2027-2037, 2013.

[31] J. Shen and J. Lam, Remarks on "Decay rate constrained stability analysis for positive systems with discrete and distributed delays", Systems Science and Control Engineering: An Open Access Journal, Vol. 3, No. 1, pp. 24-25, 2015.

[32] J. Hu, J. Liang, D. Chen, D. Ji and J. Du, A recursive approach to non-fragile filtering for networked systems with stochastic uncertainties and incomplete measurements, Journal of the Franklin Institute, Vol. 352, No. 5, pp. 1946-1962, 2015

[33] L. Wang, Y. Shen, Q. Yin and G. Zhang, Adaptive synchronization of memristor-based neural networks with time-varying delays, IEEE transactions on Neural Networks and Learning systems, Vol. 26, No. 9, pp. 2033-2042, 2015.

[34] Z. Wang, D. W. C. Ho and X. Liu, State estimation for delayed neural networks, IEEE Transactions on Neural Networks, Vol. 16, No. 1, pp. 279-284, 2005.

[35] Z. Wang, H. Wu, J. Liang and X. Liu, On modeling and state estimation for genetic regulatory networks with polytopic uncertainties, IEEE Transactions on NanoBioscience, Vol. 12, No. 1, pp. 13-20, 2013.

[36] D. Zhang, W. Cai and Q. G. Wang, Robust non-fragile filtering for networked systems with distributed variable delays, Journal of the Franklin Institute, Vol. 351, No. 7, pp. 4009-4022, 2014.

[37] S. Zhang, Z. Wang, D. Ding, H. Dong, F. E. Alsaadi and T. Hayat, Non-fragile $H_{\infty}$ fuzzy filtering with randomly occurring gain variations and channel fadings, IEEE Transactions on Fuzzy Systems, DOI: 10.1109/TFUZZ.2015.2446509.

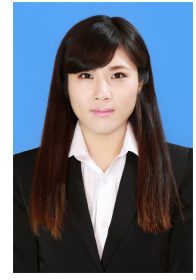

Yajing Yu received the B.E. degree in Automation in 2013 from Northeast Petroleum University, Daqing, China, and is currently studying for the M.S. degree in Control Science and Engineering from Northeast Petroleum University. Her research interests include intelligent control theory and application and neural networks.

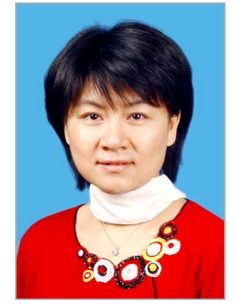

Hongli Dong received the Ph.D. degree in Control Science and Engineering in 2012 from Harbin Institute of Technology, Harbin, China. From July 2009 to January 2010, she was a Research Assistant in the Department of Applied Mathematics, the City University of Hong Kong. From October 2010 to January 2011, she was a Research Assistant in the Department of Mechanical Engineering, the University of Hong Kong. From January 2011 to January 2012, she was a Visiting Scholar in the Department of Information Systems and Computing, Brunel University, London, U.K. From November 2012 to October 2014, she was an Alexander von Humboldt research fellow at the University of Duisburg-Essen, Duisburg, Germany. She is currently a professor with the College of Electrical and Information Engineering, Northeast Petroleum University, Daqing, China.

Dr. Dong's current research interests include robust control and networked control systems. She is a very active reviewer for many international journals. 


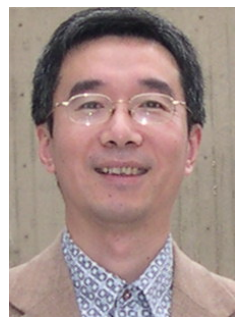

Zidong Wang was born in Jiangsu, China, in 1966. He received the B.Sc. degree in mathematics in 1986 from Suzhou University, Suzhou, China, and the M.Sc. degree in applied mathematics in 1990 and the Ph.D. degree in electrical engineering in 1994, both from Nanjing University of Science and Technology, Nanjing, China.

He is currently Professor of Dynamical Systems and Computing in the Department of Computer Science, Brunel University London, U.K. From 1990 to 2002, he held teaching and research appointments in universities in China, Germany and the UK. Prof. Wang's research interests include dynamical systems, signal processing, bioinformatics, control theory and applications. He has published more than 300 papers in refereed international journals. He is a holder of the Alexander von Humboldt Research Fellowship of Germany, the JSPS Research Fellowship of Japan, William Mong Visiting Research Fellowship of Hong Kong.

Prof. Wang is a Fellow of the IEEE. He is serving or has served as an Associate Editor for 12 international journals, including IEEE Transactions on Automatic Control, IEEE Transactions on Control Systems Technology, IEEE Transactions on Neural Networks, IEEE Transactions on Signal Processing, and IEEE Transactions on Systems, Man, and Cybernetics - Systems. He is also a Fellow of the Royal Statistical Society and a member of program committee for many international conferences.

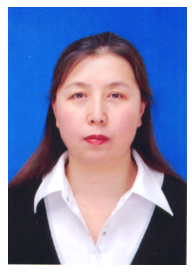

Weijian Ren received her M.S. and Ph.D. degrees from Northeast Petroleum University in Control Theory and Control Engineering in 1990 and oil storage and transport engineering in 2006, respectively. Now she is a professor at Northeast Petroleum University. Her research interests include modelling and control of complex systems, fault diagnosis and simulation.

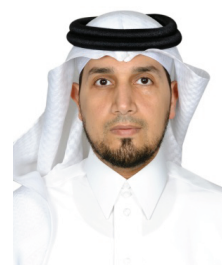

Fuad E. Alsaadi received the B.S. and M.Sc. degrees in electronic and communication from King AbdulAziz University, Jeddah, Saudi Arabia, in 1996 and 2002. He then received the Ph.D. degree in Optical Wireless Communication Systems from the University of Leeds, Leeds, UK, in 2011. Between 1996 and 2005, he worked in Jeddah as a communication instructor in the College of Electronics and Communication. He is currently an assistant professor of the Electrical and Computer Engineering Department within the Faculty of Engineering, King Abdulaziz University, Jeddah, Saudi Arabia. He published widely in the top IEEE communications conferences and journals and has received the Carter award, University of Leeds for the best $\mathrm{PhD}$. He has research interests in optical systems and networks, signal processing, synchronization and systems design. 